\title{
Sitzung vom 5. XII. 1921.
}

\section{Ein neuer Morpho. \\ Von Walter Hopp, Charlottenburg.}

Mein zur Zeit in Pasto, Südkolumbien, lebender Bruder sandte auf Grund von Schilderungen des Quellgebietes der Flüsse Caquetá und Putumayo, die ihm Indianer lieferten, einen im Fang von Schmetterlingen bewährten Indianer aus, der an bestimmten, im voraus festgesetzten Punkten dieser so gut wie unerforschten Gegend Schmetterlinge sammelte. Die ersten Sendungen aus dieser Sammeltätigkeit lieferten, obwohl erst zum geringsten Teil gemustert, bereits den Beweis, dafs dieses Gebiet eine Mischfauna kolumbianischer und ecuadorianischer mit Amazonasformen enthält, daneben aber zahlreiche ihm eigentümliche Elemente. Morpho ist bisher mit acht Arten vertreten, und zwar Formen von adonis Cr., rhetenor Cr., theseus, melacheilus Stdgr, didius Hopff., deidamia Hbn., achilles L. und hecuba L., der letztgenannte mit einer neuen, ausgeprägten Subspecies, die einen Übergang zwischen phanodemus Hew, zu hecuba darstellt und somit erweist, dafs der blauschimmernde phanodemus nicht eine selbständige Art, sondern eine Subspecies von hecuba sein dürfte. Hecuba stammt typisch aus den Guyanas; der Amazonas liefert einige ihm sehr nahestehende Formen. Als nächstes Glied schliefst sich polyidos Fruhst. von Venezuela an, dem wiederum die vorliegende Subspecies vom Quellgebiet des Putumayo folgt, während phanodemus $1000 \mathrm{~km}$ davon entfernt, an der Mündung des Putumayo in den Amazonas vorkommt.

\section{Morpho hecuba subsp. werneri nov.}

$\sigma^{7}$. Flügelspannung $132-141 \mathrm{~mm}$. Zeichnung oberseits wie unterseits nicht verschieden von phanodemus. Färbung dagegen stark abweichend, und zwar fahl grünlich weifs, also von derselben Farbe, die bei hecuba hecuba das Wurzelfeld der Hinterflügel hat. Auf den Vorderflügeln geht diese Farbe der Mittelbinde costalwärts in eine opalisierende, bräunliche Tönung über. Aufsenrand der vier Flügel tiefer sammetschwarz, breiter und fast noch schärfer abgesetzt als bei phanodemus. Auf der Unterseite alles Schwarz der Zeichnungen dunkler und mehr grauschwarz (phanodemus braunschwarz).

․ $150 \mathrm{~mm}$. Es steht der subsp. polyidos Fruhst. von Venezuela (Type 1 o, Coll. Staudinger, Berl. Zoolog. Mus.) nahe, unterscheidet sich aber wie folgt: Bei polyidos ist die Mittelbinde der Vorderflügel intensiver orangebraun (werneri: gelblich graubraun), an der Costa fast so intensiv wie von hecuba typ., reicht 
auch wie bei letzterer durchaus bis zur Costa. Bei der vorliegenden Unterart reicht die Binde nicht bis zur Costa, lärst aber dort einen dreieckigen, bräunlichen Apikalfleck stehen, genau wie phanodemus. Polyidos trägt auf der mittleren Costalfläche der Hinterflügel (wie hecuba) einen orangebraunen Wisch als Fortsetzung der Mittelbinde der Vorderflügel; bei der vorliegenden Subspecies sind dagegen die Hinterflügel ohne die geringsten Spuren von orange, rein grünlichweils.

Guagzayaco (Quellflufs des Putumayo), Südkolumbien, 1. April 1921. Type $1 \sigma^{7}$, dem Zoolog. Museum Berlin zugeeignet. Co-

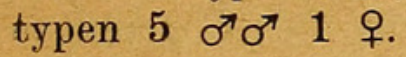

\section{Rezensionen und Referate.}

An dieser Stelle finden im allgemeinen nur Besprechungen von Büchern Aufnahme, die der Schriftleitung zur Besprechung in dieser Zeitschrift eingesandt wurden.

Willy Ramme, Orthopterologische Beiträge. Mit 3 Tafeln und 17 Textfiguren in: Archiv f. Naturgesch. Berlin, 86. Jahrg., 1920, Abt. A, 12. Heft, S. 81-166.

Eine überaus erfreuliche, sehr dankenswerte Bereicherung erfährt unsere Kenntnis der europäischen Geradflügler durch diese neueste Arbeit $\mathrm{Rammes}$. Von den 9 Abhandlungen, die sie enthält, sind besonders wichtig und mögen an ẹrster Stelle besprochen werden: Nr.I. Stauroderus (Stenobothrus) mollis Charp. - eine gute Art! und Nr. III. Ectobia lapponica L. und ihre Verwandten. Eine kritische Studie.

Durch sorgfältige Beobachtung in der Natur ist es dem V e rf. gelungen, eine verschollene Art der mitteleuropäischen Orthopterenfauna, den kleinen Grashüpfer Stenobothrus (Gryllus) mollis, den $\mathrm{Ch}$ a rpentie r vor 96 Jahren beschrieben und von dem naheverwandten St. biguttulus L. unterschieden hat, wieder ans Licht zu ziehen. Nur Yersin hat ihn beachtet und vor allem den sehr charakteristischen Zirpton des o" zur Unterscheidung von den Verwandten hervorgehoben, alle andern späteren Autoren wie $\mathrm{Fieber}, \mathrm{Fischer}, \mathrm{Brunner}$ warfen ihn mit biguttulus zusammen. Nachdem Verf. die Art in der Mark durch den charakteristischen Zirpton des $\sigma^{\gamma}$ festgestellt hatte, war es ihm möglich, die diesbezüglichen Typen $\mathrm{Ch}$ a r p e n ti e r s ( $\left.2 \sigma^{7} \sigma^{7}\right)$ in der Sammlung des Berliner Museums aufzufinden und so die richtige Bestimmung $\mathrm{zu}$ sichern. Diagnose und ausführliche $\mathrm{Be}$ schreibung in Verbindung mit den photographischen Abbildungen beider Geschlechter auf Tafel I lassen die Art, die in Mitteleuropa verbreitet ist ( $R$ ef. hat sie auch in der Gegend von Wien auf 


\section{$2 \mathrm{BHL}$ Biodiversity Heritage Library}

Hopp, Walter. 1921. "Sitzung vom 5. XII. 1921. Ein neuerMorpho." Deutsche entomologische Zeitschrift 1921(4), 296-297. https://doi.org/10.1002/mmnd.48019210418.

View This Item Online: https://www.biodiversitylibrary.org/item/103238

DOI: https://doi.org/10.1002/mmnd.48019210418

Permalink: https://www.biodiversitylibrary.org/partpdf/236275

\section{Holding Institution}

Harvard University, Museum of Comparative Zoology, Ernst Mayr Library

\section{Sponsored by}

Biodiversity Heritage Library

\section{Copyright \& Reuse}

Copyright Status: Public domain. The BHL considers that this work is no longer under copyright protection.

This document was created from content at the Biodiversity Heritage Library, the world's largest open access digital library for biodiversity literature and archives. Visit BHL at https://www.biodiversitylibrary.org. 